\title{
The Application Scenarios and System Composition of the Intelligent Convention Cloud
}

\author{
Guoyi Yang ${ }^{1}$ Ali Dai $^{2}$ Jiankang Zhang ${ }^{3 *}$ \\ 1,3 School of International Economy \&Tourism Management, Zhejiang International Studies University, Hangzhou, \\ Zhejiang 310023, China \\ ${ }^{2}$ School of English Studies,Zhejiang International Studies University, Hangzhou, Zhejiang 310023, China \\ *Corresponding author. Email: zhangjk@zisu.edu.cn
}

\begin{abstract}
Intelligent conventions, dealers conventions, company annual meetings, party building conventions and mobile conventions are the main application scenarios of intelligent convention cloud.Intelligent convention application scenarios adopt intelligent convention services,AR/VR,face recognition,indoor navigation,big data and other technologies. The application scenarios of dealersconventions adopt the one-stop and whole-process digital service of the dealer,receiver management system, hotel room distribution management system,on-site registration and check-in system,on-site ordering system,etc. The application scenarios of the annual meetings adopt the technologies such as interactive management system, annual meeting digital service system and so on. The application scenarios of the party building conventions adopt the technology of cloud convention and cloud video system. Mobile convention application scenarios adopttechnologies such as mobile

teleconference and mobile video convention system.Intelligent convention cloud continues to deepen in terms of business division, service experience, logistics management and system construction, integrating intelligent thinking into the information management of the exhibition, and constantly enriching the application scenarios to meet the needs of the development of new forms of conventions.
\end{abstract}

Keywords: intelligent convention cloud, application scenario, system composition

\section{INTRODUCTION}

The development of intelligent technology has been having a subversive impact on all walks of life,and traditional convention forms are also changing. Intelligent technological means based on information and intelligence are adopted in large conventions to create a new form of them and realize the integrated development of researching,marketing,implementation and service. The large-scale adoption of big data,AR/VR,block chain and other technologies makes the convention operation convenient,fast and efficient to a large extent,providing strong technical support for the application of intelligent convention cloud.

\section{THE CONCEPT OF INTELLIGENT CONVENTIONS}

The universally accepted meaning of intelligent convention is based on information technology, relying on cloud computing,big data analysis and the mobile Internet,with the purpose of improving the satisfaction of convention participants and efficiently managing,forecasting, and conventions[1].The practice is jointly led by the government and the market,which is the highly information-based and data-based technical system of the management of people,things, time and space resources of convention participants, as well as the management mode of systematization,rational sharing and interaction of convention market resources and social resources.

\section{THE COMPOSITON OF THE INTELLIGENT CONVENTION SYSTEM}

The intelligent convention system consists of intelligent convention service,big data,face recognition,AR/VR and indoor navigation.

\subsection{Intelligent Convention Service}

Intelligent conventionservice refers to the intelligent control of convention-related affairs management and venue equipment through the construction of the system,so that tedious tasks can be achieved with a simple operation management system, and the work efficiency can be improved. At the same time,conventions can be paperless,electronic, automated,intelligent,one-stop service and so on. The intelligent conventionservice not only saves the cost but also greatly reduces the time of participants,so 
as to achieve intelligence and efficiency,reduce the error rate, and make the convention affairs more intelligent. Intelligent conventionservice include three parts: paperless convention system,electronic convention system and intelligent convention automation system. The intelligent paperless convention system refers to an intelligent convention interaction system based on mobile Internet,cloud computing and information interaction technology. Adopting Internet technology to transform documents into electronic form, from transmission to display and then to editing embodies the networking,electronic and intelligence of the paperless convention system. [2]The flat-panel terminal of the paperless convention can be adopted for customers to view the list of participants,text chat,browse conventionmaterials, annotate and save documents, as well as watchvideo,real-name (anonymous) vote,convention vote,electronic whiteboard and so on. In addition,there are liquid crystal lifting convention terminals, desktop convention terminals,flip liquid crystal terminals,etc. Intelligent electronic convention system refers to the convention system through the adoption of electronic auxiliary means,including convention booking system,information release system, convention check-in system and so on. Intelligent convention affairs automation system refers to the automation of convention affairs management in the meeting process. According to the specific time of the conventionin thebooking system,the central control system of the convention room adjust the air conditioner to the optimal temperature half an hour before the start of the conference(central control system) ; during the period from the entry of the participants to the beginning of the convention, the lighting intensityand background music of the venue can be intelligently controlled in real time (intelligent lighting system,intelligent music system). Open the hidden microphone in front of the seat,participants can have convention speech and discussion, and the chairman can control the microphone of all participants (convention discussion, convention voting system). [3]

\subsection{Big Data}

The convention big data platform can realize the docking of supply and demand of the convention industry. There are different demand sides in the whole convention industry chain,and there is a supply-demand relationship among them. According to the different supply-demand relationship of the convention industry,different platforms need to be built by using big data. Big datafeatures realtime, at the same time, it also has $4 \mathrm{~V}$ characteristics: Volume, Velocity,Variety, Value.

The Volume of big data provides a large enough Volume of data for intelligent conventions.No matter in the aspects of acquisition,storage, management or analysis, its scale has greatly exceeded the capacity of the traditional database software, with a massive scale of data. Cloud computing provides a storage and computing platform for these massive big data. Through analysis,optimization and management of different data sources, the results can be fed back to create greater economic benefits and social values for intelligent conventions.

Velocity of big data provides efficient and rapid access to valuable information for intelligent conventions. Big data came into being very quickly by transmitted through the Internet. During the exhibition,users can upload data by scanning the code, and these large amounts of data need to be updated and processed in time. A large number of resources in the server are used to process and calculate data,and multiple platforms for real-time analysis,so that the data update speed is fast enough,making the exhibition more advantageous.

The Variety of big data provides effectiveness analysis for intelligentconventions. Single data or single user data can not constitute big data. A wide range of data sources determines the diversity of data and provesthe validity of the data.Massive data can be collected through various softwareand platforms. After analyzing users' log data and access records,big data obtains systematic structured and obvious data,achieving effective feedback to the later stage of the intelligentconvention,collecting the most representative customer preference information for the exhibition.

The Value of big data provides strong support for intelligent conventions. Both big data and the intelligentconventions essentially belong to the same platform, and the integration and development of the two will constitute a larger platform. The real-time update of the data during the exhibition is the core embodiment of big data,and the half-dead data is insufficient to support the exhibition being undertaken,so only when the stored data and information reaches a brand-new and valuable state can the convention be intelligent and meet the needs of customers.

Big data is a new tool of the convention industry,which can provide data adoption and analysis tools for the traditional business processes of the convention industry. For example,big data can provide certain data support for statistics,curatorial and investment promotion,realize the accurate matching of exhibitors, visitors and service providers, and provide application innovation in convention business process. [4]It is particularly important that the intelligentconvention can make use of the characteristics of 5G "large broadband and low latency" to accelerate the digital progress of the exhibition,providing a good network foundation for all kinds of intelligent interactive devices in the various exhibition, and help enhance the user experience. let the overall digitization of offline scenes in the future bring more imagination space.

\subsection{Face Recognition}

Face recognition refers to a kind of biometric technology for identity recognition based on facial features. The technology of collecting people's facial image or video stream by camera,detecting and recognizing by image processor,also known as face recognition and portrait recognition. 
Intelligent conventionsadopt the face recognition function to scan the face on the spot,which can effectively identify the participants,avoid miscellaneousscenes encountered during check-intoenter orderly and quickly. It achieves multiple entry points to sign in at the same time. Not only save manpower and material resources for the exhibition,but also greatly reduce the check-in time of attendees, set aside more time in the exhibition,so that the exhibition is more efficient and more intelligent.

\subsection{AR/VR}

AR (Augmented Reality) technology "seamlessly" connects the real world with the virtual world,restoring things that can not be touched or difficult to achieve in real life, and adds the corresponding image, video and 3D model technology to superimpose and interact with the virtual world and the real world.

VR (Virtual Reality) technology is the achievement of the development of computer software and hardware technology,multimedia sensor technology,robot technology, artificial intelligence, behavioral psychology and other scientific fields. The use of computer simulations allows users to observe things in three dimensions in a timely and unrestricted manner as if they were in the scene.

$\mathrm{AR}$ and $\mathrm{VR}$ are interoperable in nature, and their commonness lies in two points,"3D" and "interaction". AR technology emphasizes the combination of real and virtual,and reproduces the real world based on optical and 3D technology. Therefore, it is more inclined to reality and rationality, and easier to be used in more serious directions,such as work, training and so on. VR technology emphasizes the sense of existence or the sense of presence,It presents the images imagined and needed by human beings through the animation rendering technology. In terms of application direction, it tends to be illusory and perceptual,and more easily applied to the entertainment direction. [5]

The scope of AR/VR technology exhibited in the intelligent convention includes: stereoscopic display technology,somatosensory interaction technology,physical simulation technology,3D full-reality technology,virtual reality technology,intelligentdressing devices,augmented reality technology,3D modeling,real-time video display and control,simulated cockpit,virtual bike roaming,dynamic seat,virtual book flipping,etc. [6]

\subsection{Indoor Navigation}

Indoor navigation refers to people in a large exhibition or shopping mall,even in a building that can still use the accurate positioning function to determine where they are and where they want to go. By using indoor navigation,we can easily find designated places of large buildings, such as designated merchants, bathrooms and so on. In the process of navigation,the realization of multiple routes, discount information,promotions and new products on the market can be seen. Under the condition of meeting users' needs, economy around the routes can be driven to promote consumption and shopping.

The intelligent convention's adoptionof the indoor navigation system, which not only brings the customer from location A to location B,but also contains great commercial value, at the same time enhance the sense of science and technology and diversify the routes of exhibitors. [7]

\section{APPLICATION SCENARIOS OF DEALERS CONVENTIONS}

\subsection{The Concept of Dealersconventions}

Dealersconvention refers to the new product release and investment attraction of each merchant,and analyze the trends of the industry on the spot,to fully display their enterprise strength to the dealers,motivating the dealers and improve their enthusiasm. Meanwhile,the new strategy,new policies and new ideas of the enterprise will be further publicized to achieve the ultimate win-win situation for the manufacturer.

\subsection{The Composition of the Dealersconvention System}

The dealersconvention system is composed of pick-up system,hotel room distribution system,on-site registration and check-in system and on-site ordering system.

\subsubsection{Pick-up system}

The pick-up system includes booking the pick-up service synchronously when filling in the registration information at the micro-station, and input the pick-up date and the flight number. The organizer can export the data at any time and arrange the personnel and vehicles of the pick-up according to the situation. When there is a large number of guests to receive at the airport/station,they can use thecheck-in way to pick up. The on-site staff only need to scan the $\mathrm{QR}$ code or input the last four digits of the mobile phone number of the participants to confirm the identity of the them and complete the check-in. In case of bad airport / railway station network, they can also switch to offline check-in with one click, and then synchronize the data when the network is normal.

\subsubsection{Hotel room distribution system}

Before the convention, the hotel room distribution system will collect the guests' accommodation needs through the digital system, and the backstage automatically allocates 
the guests to share the room,so that the guests can get faster and smoother check-in experience. At the same time, the organizers follow up the data in real time,count the number of reservations and estimate in advance to avoid the problem of temporary shortage or overbooking. The system will also analyze the data according to the check-in situation of the hotel and the food requirements of the guests,automatically generate food and beverage matching to make the hotel service achieve customers' satisfaction, and enhance the competitiveness and influence of the hotel at the same time.

\subsubsection{On-site registration and check-in system}

Electronic check-in is used for on-site check-in,which can achieve the requirements of check-in mode,error-free identity verification,fast passage and non-crowding when entering the venue. There are dozens of check-in methods,such as face recognition check-in,gate checkin, WeChat scan code check-in,fingerprint check-in,ID card check-in,etc.Through the digital check-in way,we can reshape the signing-in scene of the participants, which greatly shortens the check-in time of guests, and reduces the service difficulty of on-site staff and many tedious tasks.

In dealersconventions, enterprises tend to use face recognition in combination with the digital check-in method,mainlybecause the safe and efficient recognition effect. While quickly identifying guests and customers, it can also effectively prevent people outside the invitation list from entering the venue. In addition, the guest experience will be better,the check-in speed will be faster,and also can fully demonstrate the strength of the enterprise.The QR code check-in method is also common. At the scene,guests only need to show the QR code or invited QR code successfully received for registration, and scan with equipment such as a low-light box or iPad on the spot to identify guest and customer information. According to the generated data,certificates containing guest information can be printed and generated through the printer,and the on-site license can be completed in one second,which is convenient,fast and easy to operate.

\subsubsection{On-site ordering system}

The on-site ordering system provides a new ordering mode for the dealersconvention. The core goal of on-site ordering system is to promote the products and achieve the win-win situation of the manufacturers. the rapidity of onsite ordering also determines the quality of the dealersconvention. In the case of undertaking the high heat of the interactive link, the convention realizes the new mode of ordering, uses the function of mobile phone to place an order,displays the current ordering situation on the WeChat big screen of interactive wall,updates the commodity inventory in real time, and sorts the order amount to provide a stage for massive dealers to show verve while avoiding the old-fashioned signing of orders,situations like customers do not pay or customers go back on their promises.

\section{CONCLUSIONS}

The application scenarios of intelligent convention cloud uses modern information technology to manage all aspects of exhibition activities,relying on computer technology and software systems to convey convention information more efficiently. This provides a platform for information exchange and interaction for organizers and participants. The intelligent convention cloud continues to deepen in terms of business division,service experience,logistics management and system construction,integrating intelligent thinking into the information management of the exhibition,and constantly enriching application scenarios to meet the needs of the development of new forms of conventions.

\section{REFERENCES}

[1]Sui,L.R. Focus on the party building and promote the construction of the party building to realize the construction of the party building to complement each other[J].China Chemical Safety Association,Chinese social organization,2019(07),p.17.

[2]Du,Y.Y.Research on the Development of Intelligent Exhibition in the era of "Internet $+"[\mathrm{~J}]$.Journal of Guiyang University (Natural Science Edition),2019(02),pp.8-11.

[3]Zhang,L.Application and Prospect of VR Technology in Convention and Exhibition[J].Art science and Technology,2017(07),p.75.

[4] Yang,Y.C. Development Status and Strategies of Smart Technology in Exhibition Industry J.China Science and Technology Information,2017(16),pp.116118.

[5]Yang,Y.C. Application Research of Smart Exhibition Technology in Industry Development TrendJ.Science and Technology Innovation Guide,2017(07),pp.166-168.

[6]Cong,H.B. Zhou Liandong.Research on the Development Model and Promotion Mechanism of Smart Exhibition Industry in Ningbo J.Beijing Economy,2013(03),pp.38-39.

[7]Han,L.Y.The Application of VR Technology and Big Data in Exhibition Industry J.Economic Vision,2017(22),pp.174-175. 